\title{
High-Risk Alcohol Use and Disordered Eating Behavior Before and 1 Year After Sleeve Gastrectomy
}

\author{
Emily Wong ${ }^{1} \cdot$ Aaron Fleishman ${ }^{2} \cdot$ Amanda Brem $^{3} \cdot$ Daniel B. Jones ${ }^{2,4} \cdot$ Christina C. Wee $^{3,4}$
}

Received: 11 October 2021 / Revised: 3 December 2021 / Accepted: 13 December 2021 / Published online: 28 January 2022

(c) The Author(s), under exclusive licence to Springer Science+Business Media, LLC, part of Springer Nature 2021

\begin{abstract}
Background Evidence suggests a rise in alcohol misuse after some bariatric procedures. Whether undergoing sleeve gastrectomy raises the risk of high-risk alcohol use is unclear.

Objective To characterize the risk of high-risk alcohol use 1 year after sleeve gastrectomy and collect preliminary data on potential associations between disordered eating and high-risk drinking post-surgery.

Methods We interviewed 97 patients before and 1 year after sleeve gastrectomy and assessed for high-risk alcohol use via a modified version of the Alcohol Use Disorders Identification Test-Consumption (AUDIT-C). Eating behavior was assessed using the Three Factor Eating Questionnaire Revised-18 (TFEQ-R18).

Results The prevalence of high-risk drinking increased from $13.4 \%$ prior to surgery to $22.7 \% 1$ year after sleeve gastrectomy; $16.5 \%$ of our sample reported new high-risk drinking equivalent to an incidence of $19.0 \%$. New high-risk drinkers appeared more likely to report lower cognitive restraint scores and higher scores for emotional and uncontrolled eating at baseline and had larger improvements in disordered eating scores post-surgery although these differences approached, but did not reach, statistical significance.

Conclusion One in five non-high-risk drinkers developed new high-risk alcohol intake 1 year after sleeve gastrectomy. New high-risk drinkers appear to have greater disordered eating at baseline and reported greater improvement in eating behavior than those who did not develop new high-risk drinking. These results are consistent with the addiction transfer hypothesis postulating that some patients may replace disordered eating with alcohol misuse after sleeve gastrectomy.
\end{abstract}

Keywords Sleeve gastrectomy $\cdot$ Metabolic surgery $\cdot$ Obesity $\cdot$ Alcohol misuse $\cdot$ Alcohol use $\cdot$ Disordered eating $\cdot$ Eating behavior $\cdot$ Addiction transfer

Key Points 1 . One of the five patients who undergo sleeve gastrectomy develops new high-risk alcohol use.

2. Patients who develop new high-risk alcohol use after sleeve gastrectomy appear more likely to report disordered eating behaviors prior to surgery.

3. Patients who develop new high-risk alcohol use after sleeve gastrectomy appear to report greater improvement in disordered eating behaviors after surgery.

\section{Christina C. Wee}

cwee@bidmc.harvard.edu

1 Touro University, Nevada College of Osteopathic Medicine, 874 American Pacific Dr, Henderson, NV 89014, USA

2 Division of Bariatric and Minimally Invasive Surgery, Department of Surgery, Beth Israel Deaconess Medical Center, 330 Brookline Ave, Boston, MA 02215, USA

3 Division of General Medicine, Department of Medicine, Beth Israel Deaconess Medical Center, 330 Brookline Ave, Boston, MA 02215, USA

4 Harvard Medical School, Boston, MA, USA

\section{Introduction}

Although non-surgical treatment options exist, bariatric or weight loss surgery (WLS) has been shown to provide better success with long-term weight-loss [1]. In 2019, over 256,000 surgeries were performed [2]. Despite its success, WLS is not without risks. In particular, the Roux-en-Y Gastric Bypass (RYGB) procedure has been linked to a greater likelihood of high-risk alcohol consumption post-operatively [3-5] even among patients without prior history of misuse [6]. Heavy alcohol use can lead to serious medical consequences and other adverse risks [7]. Although prior studies show an increased risk of alcohol misuse in patients that underwent Roux-en-Y gastric bypass, few studies have explored this risk for sleeve gastrectomy which has become the most commonly performed bariatric procedure since 2014 [2]. 
Sleeve gastrectomy now comprises over $60 \%$ of the bariatric surgeries performed in the USA [2]. Nevertheless, only a few studies have examined its potential impact on alcohol habits. A large multi-institutional study by Ibrahim et al. showed an increase in alcohol use after sleeve gastrectomy and identified higher educational level and higher household income as predisposing factors to this change [8]. In contrast, a study by Coluzzi et al. [9] showed a decrease of alcohol use 1 year after sleeve gastrectomy and attributed it to strict nutritional follow-up after surgery. The "addiction transfer" model has been implicated as one mechanism underlying a rise in alcohol misuse after WLS in which obese patients whose eating is driven by hedonic response to food substitute substances such as alcohol in lieu of food $[5,10]$. However, neither study by Ibrahim's nor Coluzzin examined whether prior disordered eating played a role in their findings. Other studies, however, have shown that presurgical binge eating habits lead to increase in substance use after other weight loss surgeries [4, 10, 11]. Ghrelin, an important hormone produced in the stomach for the regulation of appetite, is altered after both RYGB and sleeve gastrectomy and has been implicated to play a role in addiction $[12,13]$.

In this context, we systematically characterized high-risk alcohol drinking and disordered eating in patients before and 1 year after sleeve gastrectomy to collect preliminary data on potential associations of baseline disordered eating and changes in eating behavior after sleeve gastrectomy with changes in alcohol intake post-surgery. Characterizing the interrelationship of sleeve gastrectomy, high-risk drinking, and underlying eating habits may inform medical decisions, risk stratification, and post-op management and surveillance of patients undergoing sleeve gastrectomy.

\section{Methods}

\section{Study Design and Data Collection}

We interviewed a cohort of patients who underwent sleeve gastrectomy before and approximately 1 year after surgery. Study subjects were systematically recruited from a single academic center in Boston, Massachusetts. The center used the prevailing National Institutes of Health $(\mathrm{NIH})$ criteria to determine eligibility for surgery namely: either a BMI of $40 \mathrm{~kg} / \mathrm{m}^{2}$ or a BMI of $35 \mathrm{~kg} / \mathrm{m}^{2}$ with a major obesity-related comorbidity. Eligible patients were age 18 or older, English speaking, willing and able to participate in a telephone survey, planning to undergo sleeve gastrectomy, and enrolled prior to their surgery. Eligible patients were sent informational letters, and verbal informed consent was obtained over the telephone. Informed consent was obtained from all individual participants included in the study. The study was approved by the. institutional review board. All procedures performed in studies involving human participants were in accordance with the ethical standards of the institutional and/or national research committee and with the $1964 \mathrm{Hel}-$ sinki declaration and its later amendments or comparable ethical standards.

Participants underwent a 10-min telephone interview at baseline and 1 year after undergoing WLS. These interviews collected demographic information such as race/ethnicity, marital status, and education and eating and alcohol intake behaviors. Medical comorbidities were extracted from medical records by trained research staff.

\section{Alcohol Use and High-Risk Drinking}

We assessed alcohol use and high-risk drinking at baseline and follow-up telephone interviews using a modified version of the Alcohol Use Disorder Identification TestConsumption or AUDIT-C [14]. The AUDIT-C is a 3-item questionnaire that is used to identify people who are highrisk drinkers or who have active alcohol use disorders. The AUDIT-C is a modified version of the validated 10 -item AUDIT [15] and performs better in identifying heavy drinkers and/or those with active alcohol dependence [14]. The AUDIT-C assesses frequency of drinking over the past year, the average amount of alcohol consumed when drinking, and binge drinking over the past year. Our study added a modified version of the third item to reflect binge drinking (5 drinks or more in men, 4 or more in women) over the past month in order to improve accuracy in reporting in the context of a telephone interview and to be more consistent with the definition of binge drinking set by the National Institutes of Alcohol Abuse and Alcoholism [16]. Patients who responded to drinking more than 1 or 2 drinks on an average day or who had at least one instance of binge drinking in the last month were then asked a modified version of the remaining seven AUDIT items related to alcohol dependency and harmful alcohol use $[15,17]$. Topics related to alcohol dependency included issues over impaired control over drinking, noticeable increase of drinking, and morning drinking. Topics related to harmful alcohol included feeling guilty after drinking, blacking out, having alcohol-related injury, and concerns about drinking by others. Scores for the AUDIT-C range between 0 and 12. Our primary outcome of high-risk drinking was defined as a score of $\geq 4$ in men and $\geq 3$ in women since these scores have a high sensitivity and specificity in identifying heavy drinking and/or active alcohol abuse or dependence.

\section{Measures of Food Cravings and Eating Behaviors}

We assessed eating behavior using the Three Factor Eating Questionnaire Revised-18 (TFEQ-R18) [18]. The 
TFEQ-R18 is an 18-item questionnaire that identifies cognitive restraint, uncontrolled eating, and emotional eating; higher scores indicate a greater display of the respective disordered eating behavior. Raw scores were divided by the highest possible score and multiplied by 100 to transform the score to a $0-100$ scale for each disordered eating category. A score of $50 \%$ or higher indicate the presence of disordered eating for the respective eating behavior. We hypothesized that patients with higher baseline scores for uncontrolled and especially emotional eating were more likely to report high-risk alcohol drinking behavior after surgery. We also hypothesized patients with greater improvements in uncontrolled or emotional eating would be more likely to develop high-risk drinking after surgery.

\section{Data Analysis}

We used descriptive statistics to characterize our sample and examine the proportion of high-risk alcohol drinkers at baseline compared to 1 year after WLS. We used Pearson $\chi 2$ tests to compare the proportion of new high-risk drinkers at 1-year post-WLS relative to baseline and the proportion of high-risk drinkers at baseline who no longer reported high-risk drinking at follow-up. We examined baseline demographics and eating behaviors between those who developed high-risk drinking versus those who did not. We also compared change in disordered eating behavior between those who developed new high-risk drinking and those who did not. We hypothesized that patients who exhibited disordered eating behavior at baseline (including being prone to emotional eating or having addiction towards food) were more likely to report high-risk drinking at follow-up and that these latter patients would also demonstrate the greatest improvement in disordered eating. All statistical analyses were conducted with $\mathrm{SAS} \circledast$ (SAS® Institute, Cary, USA).

\section{Results}

Of 198 patients eligible for interview, 113 completed the baseline interview (57\%), 4 did not undergo surgery, and 1 died before follow-up. Of 109 patients, 97 participants (89\%) completed the 1-year follow-up. The overall prevalence of high-risk drinking at baseline among the 97 subjects who

Table 1 Characteristics of participants who underwent sleeve gastrectomy overall and by high-risk alcohol use at baseline

\begin{tabular}{|c|c|c|c|c|}
\hline Baseline characteristic & Overall $(n=97)$ & $\begin{array}{l}\text { Baseline non-high-risk } \\
\text { drinkers }(n=84)\end{array}$ & $\begin{array}{l}\text { Baseline high-risk drink- } \\
\text { ers }(n=13)\end{array}$ & $p$ value \\
\hline Age, years, mean (sd) & $45.2(11.8)$ & $45.5(12.0)$ & $43.3(10.8)$ & 0.52 \\
\hline Sex, female, n (\%) & $70(72.2)$ & $61(72.6)$ & $9(69.2)$ & 0.80 \\
\hline \multicolumn{5}{|l|}{ Race, n (\%) } \\
\hline White & $57(59.4)$ & $51(61.4)$ & $6(46.2)$ & 0.70 \\
\hline Black or African American & $23(24.0)$ & 19 (22.9) & $4(30.8)$ & \\
\hline Hispanic/Latinx & $15(15.6)$ & $12(14.5)$ & $3(23.1)$ & \\
\hline Other & $1(1.0)$ & $1(1.2)$ & $0(0.0)$ & \\
\hline BMI, kg/m2, median (q1-q3) & $44.1(41.4-48.1)$ & $44.2(41.7-48.1)$ & $41.9(40.0-44.6)$ & 0.13 \\
\hline Education, n (\%) & & & & 0.85 \\
\hline High school diploma or less & 19 (19.6) & $16(19.0)$ & $3(23.1)$ & \\
\hline Some college, 2-year degree & $26(26.8)$ & $22(26.2)$ & $4(30.8)$ & \\
\hline 4-year college or more & $52(53.6)$ & $46(54.8)$ & $6(46.2)$ & \\
\hline \multicolumn{5}{|l|}{ Comorbid conditions, $\mathrm{n}(\%)$} \\
\hline Cardiac or peripheral vascular disease & $9(9.3)$ & $9(10.7)$ & $0(0.0)$ & 0.22 \\
\hline $\begin{array}{l}\text { Gastroesophageal reflux disease, esophagitis, } \\
\text { Barrett's }\end{array}$ & $36(37.1)$ & $31(36.9)$ & $5(38.5)$ & 0.91 \\
\hline Psychiatric or eating disorders & $59(60.8)$ & $52(61.9)$ & $7(53.8)$ & 0.58 \\
\hline Liver disease, Cirrhosis & $26(26.8)$ & $23(27.4)$ & $3(23.1)$ & 0.74 \\
\hline Diabetes & $31(32.0)$ & $29(34.5)$ & $2(15.4)$ & 0.17 \\
\hline Other & $13(13.4)$ & $12(14.3)$ & $1(7.7)$ & 0.52 \\
\hline AUDIT-C Score, mean (sd) & $2.0(1.7)$ & $1.5(1.2)$ & $5.1(1.2)$ & $<0.001$ \\
\hline Cognitive restraint, mean (sd) & $51.2(17.5)$ & $50.8(17.3)$ & $53.2(19.1)$ & 0.65 \\
\hline Uncontrolled eating, mean (sd) & $37.5(22.0)$ & $37.9(21.7)$ & $34.9(25.1)$ & 0.66 \\
\hline Emotional eating, mean (sd) & $46.7(32.8)$ & $48.0(33.1)$ & $38.5(30.0)$ & 0.33 \\
\hline
\end{tabular}

Includes all participants who completed pre- and post-assessments 
underwent surgery and completed the 1-year follow-up was $13.4 \%(n=13)$. Table 1 presents the baseline characteristics of study participants who underwent sleeve gastrectomy overall and by high-risk alcohol use at baseline. The majority of our sample was white women with a college degree or more. Baseline high-risk alcohol drinkers had numerically higher scores for uncontrolled and emotional eating behaviors, but these differences were not statistically significant.

Includes all participants who completed pre- and post-assessments.

Figure 1 presents patients' high-risk alcohol drinking status before and after surgery among those who completed the baseline and 12-month follow-up interviews. Overall, 22.7\% of the sample reported high-risk drinking at follow-up. More than half of baseline high-risk drinkers (7 of 13) reported resolution of their high-risk drinking, whereas $16.5 \%$ of the overall sample or $19.0 \%$ of non-high-risk drinkers at baseline reported new high-risk drinking. Because the COVID19 pandemic may have altered alcohol use, we repeated our analyses stratifying by whether subjects completed their follow-up assessment before March 1, 2020; among those who completed follow-up interviews before March 1, 2020, 7 of 56 or $12.5 \%$ non-high-risk drinkers at baseline developed new high-risk drinking (see Fig. 1b).

Table 2 presents baseline AUDIT-C and disordered eating behaviors scores according to patients' high-risk alcohol use status after sleeve gastrectomy. Patients who developed new high-risk drinking appeared to be more likely to report lower cognitive restraint scores and higher scores for emotional and uncontrolled eating at baseline. New high-risk drinkers post-sleeve gastrectomy were also more likely to report larger improvements in disordered eating scores; new high-risk drinkers had a 31-point reduction in scores reflecting uncontrolled and emotional eating at follow-up, whereas scores in patients who did not report new highrisk drinking after surgery only improved by approximately 15 and 22 points, respectively. Of note, these differences approached, but did not reach, statistical significance. Similarly, mean change in eating scores among the 7 patients whose high-risk drinking appears to resolve post-surgery
Fig. 1 High-risk drinking groups 1-year follow-up relative to baseline in the overall sample (a), among those who completed the follow-up interview before (b), and after (c) March 1, 2020 (onset of the COVID-19 pandemic)

A $\quad \mathrm{N}=97$

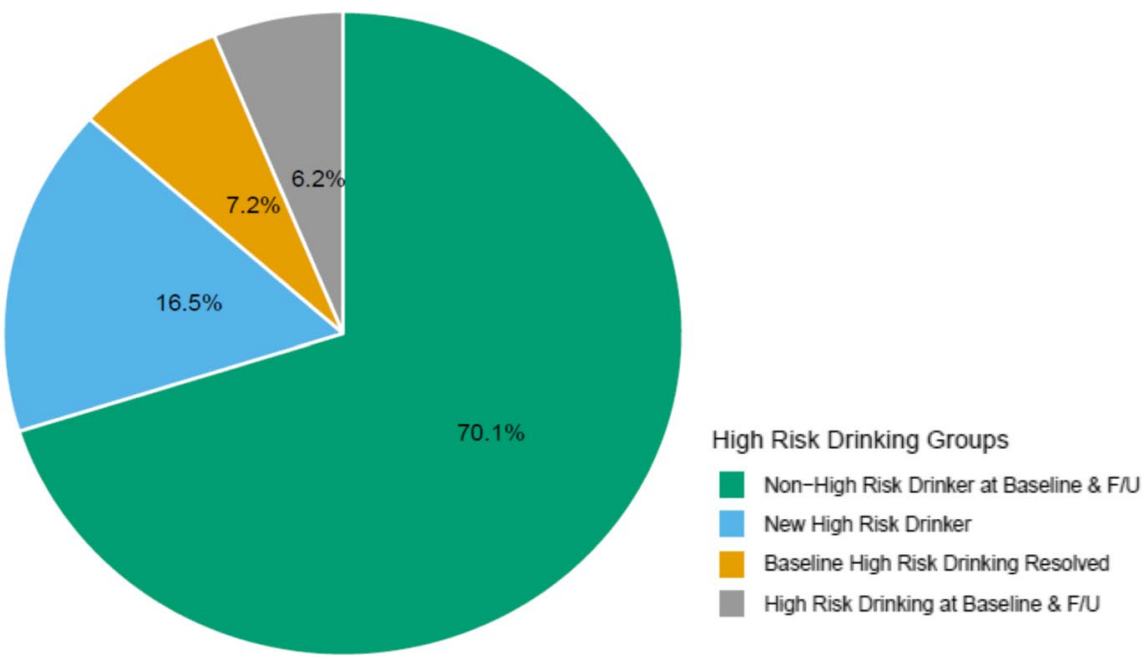

B $\quad \mathrm{N}=66$

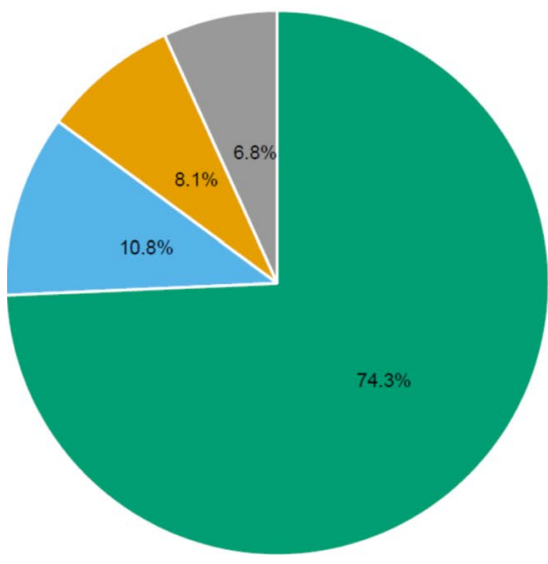

C N=22

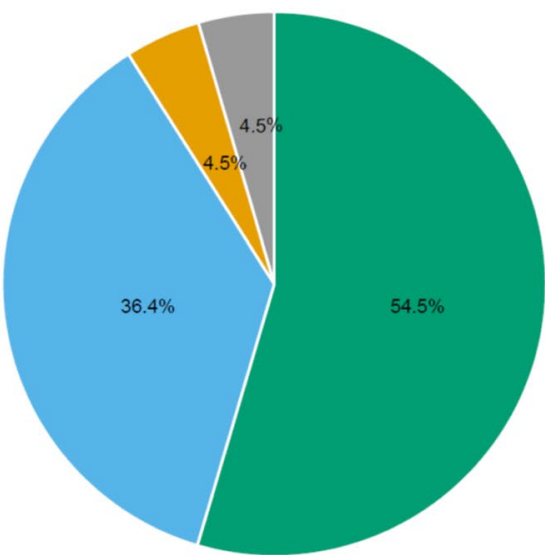


Table 2 Baseline, follow-up, and change in alcohol use and eating behavior scores across subgroups of participants based on change in high-risk alcohol use status after sleeve gastrectomy

\begin{tabular}{|c|c|c|c|c|c|c|}
\hline & \multicolumn{2}{|c|}{ Baseline non-high-risk drinkers } & \multirow[t]{2}{*}{$p$ value } & \multicolumn{2}{|c|}{ Baseline high-risk drinkers } & \multirow[t]{2}{*}{$p$ value } \\
\hline & $\begin{array}{l}\text { Remained } \\
\text { Non-high-risk drinker } \\
(n=68)\end{array}$ & $\begin{array}{l}\text { Developed } \\
\text { New high-risk drinking } \\
(n=16)\end{array}$ & & $\begin{array}{l}\text { Persistent } \\
\text { High-risk drinker } \\
(n=6)\end{array}$ & $\begin{array}{l}\text { High-risk } \\
\text { drinking } \\
\text { resolved } \\
(n=7)\end{array}$ & \\
\hline \multicolumn{7}{|l|}{ Baseline scores } \\
\hline AUDIT-C Score, mean (sd) & $1.3(1.2)$ & $2.4(0.8)$ & 0.001 & $5.2(1.6)$ & $5.0(0.8)$ & 0.81 \\
\hline Cognitive restraint, mean (sd) & $53.2(16.9)$ & $40.3(15.6)$ & 0.008 & $48.7(18.8)$ & $57.1(20.0)$ & 0.45 \\
\hline Uncontrolled eating, mean (sd) & $36.0(21.2)$ & $45.8(22.5)$ & 0.10 & $41.4(29.9)$ & $28.4(19.7)$ & 0.40 \\
\hline Emotional eating, mean (sd) & $44.9(32.1)$ & $61.1(35.1)$ & 0.079 & $48.1(32.7)$ & $30.2(27.0)$ & 0.30 \\
\hline \multicolumn{7}{|l|}{ Follow-up scores } \\
\hline AUDIT-C score, mean (sd) & $1.2(1.1)$ & $4.4(0.6)$ & $<0.001$ & $4.8(1.0)$ & $3.7(0.7)$ & 0.004 \\
\hline Cognitive restraint, mean (sd) & $61.7(16.9)$ & $64.0(15.4)$ & 0.63 & $50.0(15.7)$ & $61.1(10.8)$ & 0.16 \\
\hline Uncontrolled eating, mean (sd) & $14.5(13.7)$ & $14.8(11.4)$ & 0.93 & $15.4(19.2)$ & $16.0(17.2)$ & 0.95 \\
\hline Emotional eating, mean (sd) & $29.4(27.3)$ & $29.9(19.3)$ & 0.95 & $29.6(28.7)$ & $22.2(21.3)$ & 0.60 \\
\hline \multicolumn{7}{|l|}{ Change in scores (f/u - baseline) } \\
\hline AUDIT-C score, mean (sd) & $-0.1(1.0)$ & $2.0(0.8)$ & $<0.001$ & $-0.3(1.0)$ & $-1.9(0.9)$ & 0.016 \\
\hline Cognitive restraint, mean (sd) & $8.7(22.7)$ & $24.8(22.0)$ & 0.015 & $1.3(29.1)$ & $4.0(11.3)$ & 0.83 \\
\hline Uncontrolled eating, mean (sd) & $-21.5(17.8)$ & $-31.0(25.7)$ & 0.087 & $-25.9(21.2)$ & $-12.6(14.2)$ & 0.26 \\
\hline Emotional eating, mean $(\mathrm{sd})$ & $-15.5(31.3)$ & $-31.3(24.1)$ & 0.064 & $-18.5(26.0)$ & $-7.9(18.9)$ & 0.41 \\
\hline
\end{tabular}

was numerically lower than the 6 patients whose high-risk drinking persisted.

\section{Discussion}

Approximately one out of seven in our study who underwent sleeve gastrectomy reported high-risk alcohol use at baseline. More than half of these patients subsequently reported amelioration of high-risk drinking 1 year after surgery. However, $16.5 \%$ of the overall sample or $19.0 \%$ (one in five) of non-high-risk alcohol drinkers prior to surgery reported new high-risk drinking at 1 year after sleeve gastrectomy. Participants who exhibit a greater degree of disordered eating at baseline and who reported larger improvements in disordered eating after sleeve gastrectomy appeared more likely to develop new high-risk alcohol use, findings consistent with the addiction transfer model.

Our findings are similar to those found in an earlier study by our group [6] which examined high-risk alcohol use using the AUDIT-C scores of those who underwent Roux-en-Y Gastric Bypass (RYGB) and gastric banding. Of 369 subjects, $14 \%$ of RYGB and $20 \%$ of gastric band participants were high-risk drinkers at baseline. One year after surgery, approximately $7 \%$ of RYGB and $9 \%$ of gastric band patients developed new high-risk drinking in our earlier study. The incidence of new high-risk drinking in our current study of $19.0 \%$ is notably higher but may be in part magnified by the COVID-19 pandemic which has seen a marked increase in alcohol use [19, 20]. Nevertheless, our findings differ from the one-year results of a large multi-institutional study by Ibrahim et al. which found a $9.6 \%$ prevalence of Alcohol Use Disorder (AUS) prior to sleeve gastrectomy. At 1 year, this prevalence decreased to $8.5 \%$ but increased to $14.4 \%$ at 2 years. In contrast, our study found the overall prevalence of high-risk alcohol use increased from one in seven to one in five after 1 year. Of note, the threshold for meeting AUD is much higher than the threshold for meeting our outcome of high-risk alcohol use used in our study; thus, the estimates are not directly comparable. Nonetheless, our results show differing patterns of alcohol use before and after surgery.

Our study extends the findings from prior work by examining the link between baseline alcohol use and eating disorders and high-risk alcohol use after sleeve gastrectomy. It is important to note that almost half of baseline high-risk drinkers report resolution of high-risk alcohol use postsurgery consistent with our earlier findings after RYGB and gastric banding [6]. The paradoxical relationship between baseline disordered eating behavior and reduction in this behavior with the development of high-risk alcohol use gives credence to the addiction transfer hypothesis where patients who had high predilection for emotional or uncontrolled eating before surgery may turn to alcohol in lieu of food postbariatric surgery. Given the preliminary nature of our results, future studies are needed to confirm our findings.

Our results should be interpreted in the context of the study's limitations primarily the small sample size and limited generalizability. Our small sample size also precluded 
our ability to conduct multivariable analyses; thus, confounding cannot be excluded. We were also not able to adjust for multiple testing and there is a risk of false discovery. In addition, our primary measures were self-reported, and although we assured patients that their responses would not be shared with their clinical providers, there is likely some under-reporting of undesirable behaviors. Finally, our baseline questionnaire elicited information about use and misuse in the previous year and not use in the remote past. Thus, some of our "new" cases of high-risk drinking may reflect relapse of prior alcohol abuse behavior.

\section{Conclusion}

In summary, we found that one in five patients who were non-high-risk alcohol users develop new high-risk drinking 1 year after sleeve gastrectomy. In contrast, half of high-risk drinkers pre-operatively report resolution of their high-risk alcohol use. Our study also provides preliminary evidence consistent with the addiction transfer hypothesis where patients who exhibited greater disordered eating prior to surgery and who reported greater improvement in eating behavior post-surgery also appeared to be more likely to report new high-risk alcohol use after sleeve gastrectomy.

Supplementary Information The online version contains supplementary material available at https://doi.org/10.1007/s11695-021-05847-3.

Funding The study was supported by a grant from the Center for Nutritional Research Charitable Trust, Jamaica Plain, MA.

\section{Declarations}

Conflict of interest Daniel Jones reports being on the advisory board of Allurion. The other authors report no other relevant financial conflicts of interest.

\section{References}

1. Maciejewski ML, et al. Bariatric Surgery and Long-term Durability of Weight Loss. JAMA Surg. 2016;151(11):1046-55.

2. Surgery, A.S.f.M.a.B. Estimate of Bariatric Surgery Numbers 2011-2018. June 2018; Available from: https://asmbs.org/ resources/estimate-of-bariatric-surgery-numbers. Accessed 27 December 2021
3. Suzuki J, Haimovici F, Chang G. Alcohol use disorders after bariatric surgery. Obes Surg. 2012;22(2):201-7.

4. Wiedemann AA, Saules KK, Ivezaj V. Emergence of New Onset substance use disorders among post-weight loss surgery patients. Clin Obes. 2013;3(6):194-201.

5. Woodard GA, et al. Impaired alcohol metabolism after gastric bypass surgery: a case-crossover trial. J Am Coll Surg. 2011;212(2):209-14.

6. Author 1 et al. High-risk alcohol use after weight loss surgery. Surg Obes Relat Dis. 2014;10(3):508-13.

7. Organization WH. Global status report on alcohol and health. 2018.

8. Ibrahim N, et al. New onset alcohol use disorder following bariatric surgery. Surg Endosc. 2019;33(8):2521-30.

9. Coluzzi I, et al. Alcohol consumption after laparoscopic sleeve gastrectomy: 1-year results. Eat Weight Disord. 2019;24(6):1131-6.

10. Reslan S, et al. Substance misuse following Roux-en-Y gastric bypass surgery. Subst Use Misuse. 2014;49(4):405-17.

11 Guisado Macias JA, Vaz Leal FJ. Psychopathological differences between morbidly obese binge eaters and non-binge eaters after bariatric surgery. Eat Weight Disord. 2003;8(4):315-8.

12. Liu L, et al. Structural changes in brain regions involved in executive-control and self-referential processing after sleeve gastrectomy in obese patients. Brain Imaging Behav. 2019;13(3):830-40.

13. Peterli R, et al. Metabolic and hormonal changes after laparoscopic Roux-en-Y gastric bypass and sleeve gastrectomy: a randomized, prospective trial. Obes Surg. 2012;22(5):740-8.

14 Bush K, McDonell MB, Fihn SD, Bradley KA. The AUDIT Alcohol Consumption Questions (AUDIT-C). Arch Intern Med. 1998;1998:158.

15. Babor T, Higgins-Biddle JC, Saunders JB, Monteiro MG, The Alcohol Use Disorders Identification Test: Guidelines for Use in Primary Care. World Health Organization. 2001. 2-40.

16. Alcoholism, NIAAA. Drinking Levels Defined. Available from: http://www.niaaa.nih.gov/alcohol-health/overview-alcohol-consu mption/moderate-binge-drinking. Accessed 27 December 2021

17. Kudsi OY, et al. Prevalence of preoperative alcohol abuse among patients seeking weight-loss surgery. Surg Endosc. 2013;27(4):1093-7.

18. Bond MJ, A.M., JY Wilkinson,. The measurement of dietary restraint, disinhibition and hunger: an examination of the factor structure of the Three Factor Eating Questionnaire (TFEQ). Int J Obes. 2001;25:900-6 (Nature Publishing Group).

19. Lee BP, D.J., Leventhal A, Terrault NA. Retail Alcohol and Tobacco Sales During COVID-19. Ann Intern Med. 2021;174(7):1027-9.

20. Zipursky JS, S.N., Silverstein WK, et al. Alcohol Sales and Alcohol-Related Emergencies During the COVID-19 Pandemic. Ann Intern Med. 2021;174(7):1029-32.

Publisher's Note Springer Nature remains neutral with regard to jurisdictional claims in published maps and institutional affiliations. 